\title{
Salient Grasp of Situations as a Mechanism Against Stress for Zang Liver
}

\author{
Edwin Chau-Leung $\mathbf{Y u}^{1}$
}

1 Director, InteMed HK; Honorary Professor, Hong Kong Baptist University (HKBU), Kowloon Tong, Hong Kong

*Corresponding Author: Dr. Edwin Chau-Leung Yu, Director, InteMed HK; Honorary Professor, Hong Kong Baptist University (HKBU), Kowloon Tong, Hong Kong. Email: yuchauleung@gmail.com

Received: April 15, 2019; Accepted: July 11, 2019

\begin{abstract}
In Nei Jing, the gist of "the spirit is to subsist the grasp for changes". The visual-tactile-mental coordinative processes and the poise made out of fascia sinews are mechanisms to optimize for performance. These mechanisms are described here as an external system pertaining to the Zang Liver. The mental grasp engages external processes starting with the visual-tactile infrastructure that coordinates input-output linkages with error tracking and formal interactive adjustment. Salience of the situation through perception and cognition keeps relevant attention so that responses are made for needs and challenges as well as social situations. The understanding of salience equipped with the mental-visual-tactile-mental coordinative infrastructure and cortical associative system can be correlated with Hun, along with the Zang Liver as the director spirit keeping up to salience. With the mental-visual-tactile infrastructure built towards goal directed actions, the poise and visual-tactile grasp facilitate effective coordination. In this system, gaps and losing grasp cause fright. With anxiety, grasp tension is heightened. Too many matters in mind (as in Zang Liver) to act, anxiety ensues. Basic pathophysiology of Zang Liver can be well explained in these terms.
\end{abstract}

Keywords: Chinese Medicine, Zang Liver, Pathophysiology, Visual-tactile coordination.

\section{INTRODUCTION}

Understanding of the Zang Liver in Chinese Medicine (CM) has expanded in terms of the dispersion concept and hepatosplanchnic circulation dynamics. A lot of works has been done to investigate the contribution of the Zang Liver in pathophysiology of stress. In this paper, the mental-visual-tactile coordination system is described as it relates to the Zang Liver. Its plays a significant role in stress. It re-emphasizes the importance of the classic but often omitted description of eyes as a feature in the Zang Liver. Eye and its diseases should be related to the liver [1].

\section{THE VISUAL-TACTILE SYSTEM}

During real action, the liver and gut provide a certain degree of metabolic stability. Since actual life in a variable environment is volatile and unpredictable, unceasing tasks may pose incalculable requirements for energy and resources. To reduce uncertainties whereby erratic energy costs are reduced, operational systems are evolved to place the right action for the right job at the right time. The visual-tactile system is a coordination-corrective operating system that allows a good grasp of the environment.
To start with, the visual system is used to encompass and comprehend the surrounding environment. It is a well-evolved infrastructure with input-output linkages, error tracking, and formal interactive adjustments. Particularly, during acute rapid changes, it helps to avoid any delays that mean extra costs.

The visual system starts with extracting the stimulus content of external images. The intrinsic goal essentially is to input a stimulus-variable to the brain inferred from the retinal image. Neural responses increase with contrast. Required visual reaction time would slow down with multiple stimuli and more choices. It gets faster with practice [2] with reduced conscious effort and increased automaticity of the reaction, thus saving energy.

In case of high degree of feature overlap and perceptual similarity, discrimination may call for expanded working memory capacity, exceeding which difficulty ensues [3]. Visual attention may be categorically achieved by stimulus-driven selection, irrespective of the observer's intentions, or by goal-driven directives where selection attention is deliberate [4]. Active attention needs energy and some stimuli should be responded to and others not. The salience of an item is the state or quality by which it stands out relative to its neighbors. Saliency detection is considered to be a key attentional mechanism that 
facilitates learning and survival by enabling individuals to focus, and thereby cost-effectively use the limited perceptual and cognitive resources on the most pertinent subset of sensory inputs.

Proprioception and vision go together. Simply said, the visiolocation infrastructure is built to get a good grasp of the situation. Location information is important for saliency in detection [5]. The eyes and head move with the stimulus according to attention given. Even if frequent eye and head movements should have put objects out of visual reference, object locations are still perceived in world-based coordinates [6].

Processing input from a behaviorally relevant map would reduce scattering of energy processes during endeavors. Rapid aimed limb movements depend critically on visual information. In fact, in eye-hand coordination for movements, eyes almost always start moving toward the target before the hand does. In this visual-tactile coordinative system, vision defines the trajectory and the kinematics of reaching movements, while proprioception transforms this plan into the motor commands for the arm muscles [7]. The presence of a hand at the site also enhances orientation selectivity in early visual processing, sharpening the tuning for neural orientation [8]. Effector-based mechanisms for visual enhancement support the spatiotemporal association of gaze and reach, thus optimizing vision and guiding movements. This visual infrastructure with grasp facilitation allows a person to aim and act fast accordingly.

\section{THE MENTAL VISUAL-TACTILE SYSTEM}

Anticipatory mechanisms are common physiologically in order to meet uncertainty demands reliably. From real and interpreted memories and experience, an individual has a frame to actively and proactively respond to his environment. The mental-visualtactile infrastructure is built for effective coordination of functions towards directed actions.

The visual system first adapts readily with interpretive processes to extract visual information which can be inherently ambiguous against simultaneous contextual information. With salience, the interpretative elements of the visual-tactile coordinative infrastructure are linked with power of association [9]. Ongoing scenes and reaction patterns are gathered and formed.

To envisage a mental picture of upcoming situations, an individual may form scenarios from lifetime scenes and worldviews. The brain for a cost-effective performance would use its heuristic system to make inferences from previous scenes and moments, projecting from situational similarities for effective coping and management of uncertainties like predator considerations in the old days and social contexts nowadays. In acute or momentous operations, the visual system would instantaneously map surrounding, while scenarios guide its fast adjusting and accommodative search throughout the environment with alert sharp eyes 一眼关七, to grasp and ensemble what is salient for operation. Scenarios facilitate strategy.

On the other hand, in ordinary accustomed scenes and moments, the habitual poise composition during repetitive patterned positioning is used to optimize performance at the least cost. It is a set of body operational modes set for accustomed moments, for recurrent contexts, and as such can best stand up to future similar situations. The poise would influence a pattern of recognizable posture and practice while holding, moving and acting with force and stability. Stably in semi-equilibrium between widely divergent impulses, the poise holds a readiness in supporting the body for carrying out, coping or handling issues. The body may then put up normative responses promptly with external processes or tackling behaviors by simply fine-tuning over that habitual set. Thus, gymnasts trained for Olympics remain so poised whatever mentally and physically challenging the event may be. Not only muscles tuned up. The endomysial myofascia net, as a slowly reacting but plastic system, accommodating postural patterns, is strung with repetitive use to align to the lines of tension and movement. Set points for spatiotemporal orientation would be cost saving over long-term adaptation.

\section{USEFULNESS FOR CHINESE MEDICINE ELABORATION}

The understanding of salience equipped with the mental-visualtactile-mental coordinative infrastructure and cortical associative system can be correlated with Hun 魂 through mental grasp associated with the Zang Liver.

In keeping saliency, the body and mind are focused on situations and environment. This is quite akin to the CM Hun. Hun is the kind of spirit as a director, having capacity to know. Losing Hun refers to absent-mindedness as if overtly dazzled or distracted. With salience, the mind and body can attentively grasp the situation in control. In CM, the Zang Liver houses or carries the Hun. Salience, as the Hun, keeps relevant attention, makes responses for needs and challenges. Anticipation mechanisms may initiate tackling processes to the surrounding environment. Coupled with scenarios, the prefrontal cortex holds a plan in focus for long enough duration to complete it.

CM describes further how with the Zang Liver, the Hun or the mind opens itself at the eyes. The mental-visual-tactile system provides the effective operational infrastructure towards goal directed actions. Notably, Hun operates in both top-down and bottom-up modes (mental-visual-tactile and visual-tactile-mental modes). Mastery of habitual body patterns while grasping the immediate environment succinctly is achieved when bottom-up signals and top-down signals both jointly contribute to the allocation of attentional priority [10]. Up in the brain, the prefrontal brain, pertinently situated above the eyes, would function to support effective anticipatory foresight. Former scenes and moments through life would help to foster scenarios, overcoming pertinent variations in activity. Below this, with the visual-tactile infrastructure coordinating formal interactive adjustment, together with the poise patterned by visiospatial linkage from memory and experience, the person can execute control by fine-tuning to match situations more exactly and easily. This set of composure from repetitive patterned positioning would allow situations be managed well enough.

Situation change requires a new grasp for readiness, altering habituated patterns if necessary. Whenever there is a significant change, the grip heightens for grasping, eyes are perfused, and hand are at the same time perfused [11], all being prepared for any switch needed for readjusting physical or mental grasp. CM understood that "as environment enters through the eyes [12], its essential matters are kept in Zang Liver [13], and, as genuine (life) matters are disposed with Zang Liver, it is carried in the Qi of sinew endomysium for grasp [14]". This accords well with the $\mathrm{CM}$ view that "The Zang Liver, energizer capital, carries the Hun...charging up the fascia sinews, generating Blood and Qi activities [15]." This may equate to a kind of body intelligence for operations.

Nei Jing also described that "the Liver develops sinews....and liver governs the eyes... The spirit.... subsists the sinews for the 
body, and the Zang Liver for organs... subsists the grasp for changes, the eyes as gateway [12]." CM literature illustrates how the mind, eyes, fascia sinews, brain, and grasp influence each other. As these are working in operational mode together for integral needs, it empowers a person's adaptation and actions.

\section{RELEVANCE TO STRESS}

Preparedness needing for physical or mental grasp readjustments cannot be unduly carried on and on. Neijing further described that "when essence and Qi effects build up (excessively) in the Zang Liver, anxiety ensues". Plainly interpreting this, too many active matters (mental-visual-tactile coordinative processes) for overly diverse uncertainties would result in worries. Also, too much stuffed up and not well disposed, anger prone. When eyes are closed with no more dissipative involvement, the grasp is loosened, and trade-offs from recruitment of hepato-splanchnic circulation are restored.

Short of a good match in preparedness to the situations for resource restoration, the body or mind may be tuned up, or ill tuned, or become exhausted. Generally, it is the mesolimbic dopaminergic reward system that evaluates the salience of social stimuli. Dopamine is synthesized and released specifically and principally in only some brain regions and, interestingly, also in the retina [16]. Dopamine is a key neurotransmitter that modulates the function of neural circuits supporting motor function, cognition and vision. Mood problems are mostly related to the dopamine system, and these are major constituting symptoms in Zang Liver pathologies.

While supporting for mental grasp, the hold for physical grasp may be enhanced with long-standing anticipation. Poise strungup may become generalized to serve preparedness. In fact, the most discriminative somatic symptom of generalized anxiety disorder compared to other anxiety disorders is its heightened muscle tension [17]. Prepared for a feat, the lock-in mental and physical processes would be installed for that matter. Sometimes, extraordinary events may be prolonged and the poised composure as an attempt to overcome the situations becomes exhaustive and not cost-effective.

It is common to see tightly shut mouths and clenched jaws watching exciting games or when sustaining a certain poise in a position of alertness repeatedly. Prepared for feats but not in full control or when the endeavor is not actuated, overpreparedness is dry assertion. The body provides for metabolic support and stability to activities. However, supported, metabolic constraints are certainly present. In this regard, exhaustion observed in Nei Jing described: "as the Zang Liver -Qi (machinery) is heated up, sinews working dry and tight got cramped up, ending in atrophic sinews". Prolonged exhaustion over-drains any support processes.

Body adjustments may be highly-strung but not sufficient to cater when demands are acute and serious. Efficiency requires predicting what will be needed, and prediction requires each effector to adapt its output to the expected range of demand [18]. With fast changes, the visual-tactile-mental coordinative processes in its physical body frame would become stressed, and the autonomous processes thrown out of equilibrium. The strung mental-visual-tactile composure would not suffice such changes. Nei Jing, among five Zang Organs' aversion, describes that "the Zang Liver would not tolerate Wind". Wind in CM is analogous to fast abrupt changes. CM texts describe, "fast changes (Wind), loss of grasp (Drop off), and wobbling sight (dizziness) are all related to Zang Liver". Notably, eyes are much more frequently involved in Liver - Wind dysfunction (70.4\%), compared to " Wind " problems due to other pathologies such as general Blood Insufficiency (53.3\%) or Yin Insufficiency (45.3 \%) [19].

The poise and attention can withstand only a certain ambit but not too much discrepancies and uncertainty issues. Demanding situational changes would cause rational and emotional problems. Other body processes are needed to cover gaps and insufficiencies. Glucocorticoids (GC) are raised for the inadequate maintenance of homeostasis during coping with physical and emotional needs when there are changes in drives and anticipatory adaptations. Apart from being stressresponsive in the HPA neuroendocrine axis, steroid induction of neuropeptides and neurotransmitter gene expression in functional circuits in the brain helps sustaining the central arousal states [20].

A person has to face many situations: from predator emergence or prey hunting since the old days up to intruder challenge, reproductive opportunity, food availability and problem-solving in present times. Over-stressed, in time, behavioral responses adapting and adjusting to environmental events may have conflicting motivations in the context of future needs and resource allocation. Overt discrepancies raise anger and rage. Nei Jing states "Liver-Qi...stuffed up causes angers". Cumulated long-term tradeoffs may be overdue. Socially, a visual stimulus would basically be necessary and sufficient for an aggressive response [21].

The person seeks as a whole to re-attain a good grasp of the situations by re-matching his system competency with efficiency and effectiveness. Once achieved, return of the salience will reduce demand signals, and the individual remains vigilant and cognitively competent for his environment, having a state of learned preparedness in the system. Lessons learned from one experience are transferable to the new situation and domain, through scenarios and poise. Judgment, insight, motivation and mood, are critical. Catering for situations in control, the CM Zang Liver is taken analogous to the "master general, driving out planned controls and discretion" with direction and organization. Congruence with scenarios generating plans and a poised state of optimal function can maintain virtuous cycle generation of effectiveness and efficiency to overcome interactions with the environment.

For the Zang Liver, the hypothalamus and ANS are also closely involved [22]. CM describes its capacity for regulation of snug processes in body dynamics (free coursing" [23,24]) by redistributing energy and perfusion, maintaining blood vessels open and unobstructed, regulating gastrointestinal transport, modulating emotional flows and promoting reproductive mechanisms. The dispersion function driven by Zang Liver should be ascribed to auto-regulatory processes.

For management, interruption of the cascade of autonomic pathways helps preventing depression and stress responses and diseases. Understanding the importance of the visual grasp, additional treatment may be geared to this direction. Qua-sha by scraping the skin and fascia may reduce fatigability [25] by revitalizing the sinews and microcirculation, and generally helps Zang Liver problems. Taiqi or Gongfiu training improves posture, poise and grasp as well as tackling behaviors. Besides, reducing demand to the associated visual coordinative processes would also eases tension. Restraining energy-draining eye habits like overdoing digital usage should ease overcoming stresses. Proficiency and support during life processes will attain a good grasp by matching system competency with efficiency and effectiveness to the environment. 


\section{CONCLUSION}

The mind, or in CM the director spirit Hun, keeps salience of the moves in productive performance. Tackling processes as the external set of the Zang Liver for situations comprises the mental-visual-tactile coordinative and awareness system including salience in grasp, vision, poised physiological responses, and perceptive scenarios. Congruence with scenarios generating plans and the poised state of optimal function can maintain virtuous cycles of effectiveness and efficiency to overcome interactions with the environment. With sustained cost-effectiveness, multiple diverse body functions and internal auto-regulatory processes will be in snug (and freecoursing), and the body will have increased capacity and more adaptive power for situational complexity. That is the way to overcome internal-external stress. CM describes that in any change, the gist is grasp [12]. The Chinese phrase 把握 (hold and anticipate in mastery) starts with the same word.

The mental grasp engages external processes starting with the visual-tactile infrastructure that coordinates input-output linkages with error tracking and formal interactive adjustment. Salience of the situation through perception and cognition allows relevant attention, making responses for needs and challenges as well as social situations. Coordinative machinery to match environment is done with poise and visual-tactile grasp. The poise patterned by visiospatial linkage from memory and experience acts as a base to facilitate execution through control and fine-tuning. Formed scenarios facilitate their execution, overcoming pertinent variations in activity.

Salience regulation and activation system for grasp and poise through sinews and visual-tactile-mental coordination are a series of anticipatory physical and mental function. These are related to stress reaction and formation as this is a dominant pathology in Zang Liver. In volatile and unpredictable environment, it may be difficult to buildup reliable mechanisms for living or to match capabilities with cost-effectiveness in life. If a good grasp is achieved, situational demands are well disposed, the body and mind operations are well coordinated, and life and work silently go on. Gaps can cause fright. Too many matters in mind (and in Zang Liver) to act, anxiety ensues.

Previous explanation of CM "the liver opening into the eyes" would have been viewed just as sophistications to justify CM theory. The new understanding from this paper explains the Zang Liver in better pathophysiological terms, satisfying both ancient CM and modern medicine. Later exploration of Zang Liver should take this into consideration during study.

\section{REFERENCES}

1. Deng $Y F, X u Q$, Wang $B H$ : Exploration and analysis of the liver opening into the eyes. Hunan Zhong Yi Za Zhi 2016;32:139-140.

2. Rostami HR, Ashayeri $\mathrm{H}$. Effects of motor skill practice on reaction time and learning retention in Parkinson's disease. Neurol. India, 57:768-71, 2009.

3. Ashley R. Knutson, Ramona O. Hopkinsb,c, and Larry R. Squire. Visual discrimination performance, memory, and medial temporal lobe function. Proc. Natl. Acad. Sci. USA., 109(32): 13106-13111, 2012.

4. Egeth HE, Yantis S. Visual attention: Control, representation, and time course. Annual Review of Psychology, 48, 269-297, 1997.

5. Ren T, Liu Y, Ju R, Wu G. How important is location information in saliency detection of natural images. Multimed. Tools Appl., 75:
2543-2016.

6. Cavanagh P, Hunt AR, Afraz A, Rolfs M. Visual stability based on remapping of attention pointers. Trends Cogn. Sci., 14(4): 147153, 2010.

7. Sarlegna FR, Sainburg RL.The Roles of Vision and Proprioception in the Planning of Reaching Movements. Adv Exp Med Biol., 629: 317-335, 2009.

8. Perry CJ, Sergio LE, Crawford JD, Fallah M. Hand placement near the visual stimulus improves orientation selectivity in V2 neurons. J.Neurophysiol., 113(7): 2859-2870, 2015.

9. Ludwig CJH, Gilchrist ID. Stimulus-Driven and Goal-Driven Control Over Visual Selection. J.Exp.Psychol.Hum.Percept.Perform., 28(4):902-12, 2002.

10. Wolfe JM. Guided search 4.0: Current progress with a model of visual search. In, W. Gray (Ed.), Integrated Models of Cognitive Systems, Oxford, New York, pp. 99-119 R, 2007.

11. Stewart JM, Montgomery LD, Glover JL, and Medow MS. Changes in regional blood volume and blood flow during static handgrip. Am.J.Physiol.Heart Circ.Physiol., 292: H215-H223, 2007.

12. (唐)王冰, 黄帝内经. 素问, 第二卷: 第五篇, 阴阳应象大论。

13. (唐)王冰, 黄帝内经. 素问, 第五卷: 第十八篇, 平人气象论。

14. (唐)王冰, 黄帝内经. 素问, 第三卷: 第九篇, 六节藏象论。

15. (唐)王冰, 黄帝内经. 灵枢, 第二卷: 第八篇, 本神论。

16. Jackson C R, Ruan G X, Aseem F, Abey J, Gamble K, Stanwood G, Richard D. Palmiter, P. luvone M, and McMahon D G. Retinal Dopamine Mediates Multiple Dimensions of Light-Adapted Vision. J. Neurosci., 32(27):9359-9368, 2012.

17. Pluess $\mathrm{M}$, Conrad $\mathrm{A}$, Wilhelm $\mathrm{FH}$. Muscle tension in generalized anxiety disorder: A critical review of the literature. Journal of Anxiety Disorders, 23, 1-11, 2009.

18. Sterling P. Principles of Allostasis: Optimal Design, Predictive Regulation, Pathophysiology, and Rational Therapeutics. In: Schulkin J. ed. Allostasis, Homeostasis, and the Costs of Physiological Adaptation, Washington DC: Cambridge University Press, pp.17-65, 2004.

19. 胡随瑜, 肝阳上六证实验诊断指标研究。中医杂志。1998; 39(11) :680。

20. Schulkin. Introduction, In: Schulkin J. ed. Allostasis, Homeostasis, and the Costs of Physiological Adaptation, Washington DC: Cambridge University Press, pp.1-17, 2004.

21. O'Connell LA, Rigney MM, Dykstra DW, Hofmann HA. Neuroendocrine mechanisms underlying sensory integration of social signals. J.Neuroendocrinol., Jul;25(7):644-54, 2013.

22. 突破中医藏象研究的难点——形体医理和机能医理的解钥, 2015 。 http://www.uuuwell.com/article-1246169-1.html

23. 房克英, 肝藏血主疏泄的现代文献研究。北京中医药大学硕士论 文。2013。

24. 于峥, 黄晓华, 滕静如, 杨威, 肝失疏泄的现代研究进展。中国 中医药图书 情报杂志。2014；38(5):58-62。

25. Wang Xingze, Chen Peijie, Huang Xingyu, Wang Yingying, Yang Jinsheng, Wichai Eungpinichpong, Yang Yuming, Uraiwan Chatchawan. Guasha improves the rating of perceived exertion scale score and reduces heart rate variability in male weightlifters: a randomized controlled trial. J Tradit Chin Med.; 37(1): 49$56,2017$.

26. Zhang C, Chen Y, Yang M, Wang GY, Zhou P, Kou YH, Gao HW: Explanation of the Chinese medicine theory of "the liver opening into the eyes" from the modern medicine. Shizhen Guo Yi Guo Yao 2009;20:233-234. 